\title{
Equidad y distribución geográfica de recursos financieros en los sistemas de salud
}

\author{
Equity and geographic distribution of financial \\ resources in health systems
}

Silvia Marta Porto 1

\footnotetext{
1 Departamento de Administração e Planejamento em Saúde, Escola Nacional de Saúde Pública, Fundação Oswaldo Cruz. Rua Leopoldo Bulhões 1480, Rio de Janeiro, RJ 21041-210, Brasil. sporto@ensp.fiocruz.br
}

\begin{abstract}
This study focuses on equity in health and specifically the geographic distribution of financial resources. The author reviews the main contemporary theories of social justice and discusses the concept of equity in general and specifically in the health field. Based on the discussi on of selected international experiences (United Kingdom, Spain, and Italy), the Resource Allocation Working Party (RAWP) formula used in the United Kingdom is identified as the most adequate distributive methodol ogy, si zing the rel ative needs based on the population's demographic and epidemiological profiles. Finally, the results are presented from a simulation performed for the Brazilian case, showing that a more equitable geographic distribution of financial resources would requi re a redistribution favoring the States of the North and Northeast. The article concludes by highlighting that a comparison of actual fund outlays by the Ministry of Health in 1994 and the results of the simulation with the RAWP methodology for the Brazilian case show that the principles written into Brazilian legisl ation were absent from the geographic distribution of financial resources.
\end{abstract}

Key words Equity; Health System; Health Services; Health Policy

Resumen Este trabajo aborda la temática de la equidad en la salud, pero específicamente, en la distribución geográfica de los recursos financieros. Hace un repaso de las princi pales teorías de justicia social en el pensamiento contemporáneo y discute el concepto de equidad en general y en el campo dela salud. Tomando como base la discusión de algunas experiencias internacionales (Inglaterra, España e Italia), se identifica como metodol ogía distributiva más adecuada la fórmula RAWP Ilevada a cabo en Inglaterra, que proporciona dimensiones a las necesidades relativas a partir de los perfiles tanto demográficos como epidemiológicos de la población. Finalmente se presentan los resultados obtenidos en simulación efectuados para el caso brasileño. Los resultados presentados para el caso brasileño permitieron verificar que, para una distribución geográfica de recursos financieros más equitativa, sería necesaria una redistribución en favor de los estados de las regiones Norte y Nordeste. Final mente se destaca que, la comparación entre la utilización de recursos efectuada en 1994 por el Ministerio de Salud y los resultados obteni dos en la simulación de la metodol ogía RAWP para el caso brasileño muestran que los princi pi os enunciados en la legislación no estuvieron presentes en la distribución geográfica de recursos financieros.

Palabras clave Equidad; Sistema de Salud; Servicios de Salud; Política de Salud 
Introducción

La búsqueda de justicia, en general, y de la justicia social, en particular, han sido objeto de preocupación permanente, de la misma forma que la temática de la equidad está ocupando un lugar de relevancia tanto en la discusión teórica acerca de la justicia como en la realización de las políticas sociales. Entre los principios en los que se orientan las políticas sociales, en general, y las políticas de salud, en particular, la equidad es uno de los que ha alcanzado mayor consenso, en especial en las últimas décadas. No obstante, en muchos casos, las políticas implementadas no garantizan su ejercicio efectivo, terminando por restringir su validez a la definición formal de un derecho.

Entre los distintos aspectos que abarca la temática de la equidad en salud, se vuelve aquí, como tema central, a la equidad en la distribución geográfica de recursos financieros en los sistemas de salud.

Por ello, este artículo se propone:

- Sintetizar las distintas teorías de justicia social en el pensamiento contemporáneo y discutir el concepto de equidad en general y de salud en particular, así como su aplicación en la distribución de recursos financieros en los sistemas de servicios de salud.

- Analizar la experiencia de algunos países que llevaron a cabo metodologías orientadas por el objetivo de la equidad para la distribución de recursos financieros en los respectivos sistemas de salud, tales como las propuestas elaboradas en Inglaterra, España e Italia.

- Presentar los resultados de simulaciones efectuadas a partir de la metodología inglesa aplicada al caso brasileño, comparándose los resultados obtenidos así con la repartición efectuada por el Ministerio de Salud (MS), en el año 1994.

Se concluye que la equidad en los sistemas de salud de los países estudiados está legalmente definida por la igualdad de oportunidad de acceso para necesi dades iguales, sin embargo no siempre la operacionalidad de este concepto desemboca en mejores niveles de equidad. Se destaca que, la comparación entre la distribución efectuada en 1994 por el MS y los resultados obtenidos en la simulación de la metodología Resource All ocation Working Party (RAWP) para el caso brasileño muestran que los principios enunciados en la legislación no estuvieron presentes en la distribución geográfica de recursos financieros.
J usticia social y equidad en salud

Teorías de justicia social en el pensamiento contemporáneo

El punto central que ha servido como pauta en la elaboración de propuestas de justicia social en el pensamiento contemporáneo está relacionado con la necesidad de definir qué igualdad se busca, qué desigualdades son aceptables y cuáles serían, inclusive, deseables (Culyer, 1988; Freurbaey, 1995b; Klein, 1988; Pinker, 1988; Rawls, 1985; Sen, 1992).

En este sentido, se destaca la teoría de la justicia desarrollada por Rawls (1985). Hasta la formulación efectuada por este autor, las contribuciones en este campo de conocimiento se limitaron a citar ejemplos de injusticias permitidas en la aplicación de la concepción teórica utilitarista, según la cual una sociedad está correctamente ordenada y, por tanto, es justa, cuando obtiene la mayor suma de satisfacción, independientemente de su distribución, entre los individuos pertenecientes a esa sociedad. De esta forma, se justifica la penalización y exclusión de individuos, teniendo como meta la obtención de mayor suma de satisfacción, cuyos beneficios, en última instancia, serán apropiados por aquellos que se encuentren en situación favorable al aumento de la utilidad marginal que será obtenida. La maximización de la satisfacción es producto natural del libre actuar de los individuos y de los mercados. De este modo, se puede afirmar que, para los utilitaristas, la igualdad deriva exclusivamente de la libertad y por tanto adquiere dimensión estrictamente formal en sus formulaciones.

Así, intentando contraponerse al pensamiento utilitarista, Rawls (1985), que entiende la sociedad como un sistema cooperativo cuyo objetivo es la obtención de ventajas mutuas, propone partir de una hipotética posición inicial, sin realidad histórica, en la que sería establecido un contrato “... ya no como resultado de una opción política, sino como una discusión de una cuestión filosófica" (Lacoste, 1992:138).

En la posición inicial, las personas libres e iguales deberán abandonar simpatías y odios, colocándose en situación de imparcialidad, denominada por el autor "velo de la ignorancia", en la que desconocen tanto su posición social original como sus capacidades y preferencias, pero conocen los principios de la teoría económica, las cuestiones políticas, las bases de la organización social y las leyes de la psicología humana. Según Rawls, las personas libres, iguales y racionales, asumiendo el "velo de la ignorancia” como punto de partida, llegarían a de- 
finir, en la carta fundadora de su asociación, los siguientes principios:

1) “... cada persona debetener un derecho igual al sistema más amplio posible de libertades bási cas; todos I os socios ti enen derechos a la más extensa libertad compatible con la libertad de los demás"

2) "Las desigualdades económicas y sociales deben ser tales que:

a) sirvan al mayor beneficio delos más desfavorecidos, dentro delos límites de un justo principio derepartición;

b) deben estar relacionadaslasfunciones y posiciones abiertas a todos, deacuerdo con el principio de justa igualdad de oportunidades" (Rawls, 1985:82).

Los principios mencionados deberán leerse en el orden presentado, con lo que queda claramente expuesta la prioridad dada a la libertad. Es en este aspecto que no son admisibles las desigualdades entre los individuos. El segundo de los criterios, que trata de la forma de distribución de la renta, de la riqueza, y de las grandes líneas de organización, por un lado, pretende disminuir las desigualdades por medio de la elección de una política "maximin" (maximizar el beneficio de los menos favorecidos) y, por otro, manifiesta la aceptación de la desigualdad entre los hombres, siempre que se entienda que son justas.

Estos principios de justicia son los que deberán ser utilizados para la distribución de los “bienes primarios", definidos por el autor como los tipos de bienes que todos los individuos prefieren tener más que menos, por ejemplo: riqueza, posición social, oportunidades, habilidades, libertad, y hasta incluso auto-respeto.

Para Rawls (1985), la garantía de que los principios escogidos serán equitativos está determinada por las circunstancias especiales de constitución de la posición original, en la que deben existir relaciones simétricas entre las partes. En otras palabras, Ios resultados equitativos están asegurados por la característica equitativa de la situación inicial. Sin embargo considera que para la efectiva realización de los criterios de justicia es necesaria la intervención gubernamental a través de sus instituciones, en el sentido de asegurar un mínimo social, a partir de la distribución de los bienes primarios (Rawls, 1985).

No hay duda de que estos son los aspectos innovadores que han transformado la teoría de la justicia elaborada por Rawls en el objeto de análisis de innúmeros trabajos.

Las opiniones acerca de la obra compleja y ambiciosa de Rawls fueron extremadamente diversas. Algunos autores, como Stuart Hamps- hire, Warnosck y Marshall Cohen, calificaron el trabajo de "magistral", afirmaron que se trataba de una refutación a la acusación de que la filosofía analítica no puede contribuir a un pensamiento moral y político substancial y entendieron la propuesta rawlsiana en toda su amplitud (Gorovitz, 1979). Otros, a pesar de que reconocieron su importancia, consideran el trabajo de Rawls, incoherente, confuso y todavía marcado por el pensamiento utilitarista.

A pesar de las críticas, la teoría de la justicia de Rawls (1985) aporta bases fundamentales a la búsqueda de una conceptualización del término equidad. Basta destacar la incorporación de intereses colectivos como resultado del contrato original entre individuos, el papel del Estado en la distribución de los bienes básicos y la preocupación con la disminución de las desigualdades por mediación de políticas que, en alguna medida, mejoren la situación de los menos favorecidos.

Otra contribución importante en el campo de la justicia social es la elaborada por Sen (1992), que asume, como valores centrales, la igualdad y la libertad, pero entendiéndose esta última en sentido amplio, en el cual, además de la posibilidad de elección individual, se incluye el requisito de seguridad social y económica.

Sen considera que la vida puede ser vista como un conjunto de funciones interrelacionadas y que las realizaciones, o resultados, personales pueden ser entendidas como el vector resultante de esas funciones. Como ejemplo de funciones relevantes, se menciona estar bien nutrido, tener buena salud, así como algunas más complejas, como ser feliz, tener auto-estima (Sen, 1992). Sin embargo, el punto central, según este autor, está en la capacidad de que los individuos ejerzan esas funciones, según la capacidad que tengan las personas de transformar bienes en funciones. El conjunto de capacidades del individuo representa su efectiva oportunidad de consecución del bienestar, asegurando la libertad de elección entre formas de vida - elección entre el conjunto de vectores de funcionamientos posibles (Sen, 1992).

La igualdad en la distribución de los bienes primarios propuesta por Rawls, en la comprensión de Sen, no es suficiente para asegurar tratamiento equitativo, pues la desigualdad de capacidades es el principio primordial de ajuste para la obtención de la equidad.

Importa distinguir capacidades de bienes primarios y de realizaciones; por ejemplo, una persona puede tener más bienes primarios que otra, medidos tanto en recursos como en libertad, pero ser portadora de algún tipo de incapacitación, o incluso dos personas pueden te- 
ner igualdad de capacidades, pero llegarán a estados de bienestar diferentes simplemente como resultado de la libertad de elección (Sen, 1992).

En esta propuesta, el estado de salud depende de la capacidad de funcionar que las personas tienen a su disposición, incorporando, a este concepto de "capacidad", diferentes factores determinantes de las necesidades tanto los relacionados con las características biológicas y sociales de los individuos como los derivados de la oferta existente, producto de las políticas sociales puestas en marcha, incluyendo variables referentes a la calidad de los servicios. O sea, tal capacidad es determinada por los bienes - por ejemplo, cuidados de salud, educación, alimentación - y por las características de los bienes - eficacia clínica, conocimientos, composición proteica de la dieta. Asimismo, las características de los bienes se encuentran conectados a los factores ambientales - disponibilidad de cuidados médicos, de buena educación, de alimentación nutritiva - y las características personales - edad, nivel socio-económico, dimensión de la familia.

Basándose en lo expuesto, se puede afirmar que, para Sen, la equidad debe ser entendida como igualdad de oportunidades y que, para su efectiva consecución, es necesario compensar las desigualdades de capacidad.

Finalmente, se destaca la contribución realizada en los últimos años por Fleurbaey (1995), para lo que importa la obtención de “igualdad de realizaciones fundamentales". Por tanto, se propone hacer una lista con todas Ias realizaciones expresivas para los individuos, indicando, en cada caso, si el resultado es responsabilidad personal o social.

Para el conjunto de realizaciones de responsabilidad social, denominado "Equalisanda" - entre las cuales, según el autor, se encuentra la salud - deben ser definidos los recursos distribuibles, los talentos o handicaps que no son redistribuibles, pero justifican la intervención pública, los resultados esperados - que pueden ser entendidos como las funciones de Sen; los centros de decisión responsables por las realizaciones - gobierno, autoridades locales, empresas públicas; y el ámbito de ejercicio de las responsabilidades, por ejemplo, respecto a la distribución de los recursos o a los resultados (Fleurbaey, 1995a, 1995b).

Esta forma de abordar el asunto conserva los aspectos interesantes de las teorías contemporáneas mencionadas; el acento no está puesto en los recursos en sí mismos, sino en lo que ellos permiten hacer (Sen), pero limitando lo que forma parte del concepto de igualdad
(Rawls), referida en este caso a las realizaciones fundamentales de responsabilidad social.

De esta forma, se puede decir que la contribución del pensamiento contemporáneo se centra en la valorización de la noción de igualdad como objetivo final, sea de bienes primarios, de oportunidades y condiciones (capacidades) sea de realizaciones fundamentales, así como en el reconocimiento explícito de factores determinantes de las diferencias existentes, que están relacionadas con aspectos biológicos, sociales y político organizacionales. En otras palabras, asume la necesidad de asegurar derechos igualitarios a parte de las diferencias individuales.

Finalmente se destaca que, incluso reconociendo la importancia de la contribución efectuada por Rawls, en su Teoría dela Justicia, es el aporte de la concepción elaborada por Sen, fundada en el concepto de“capacidades básicas" y con el entendimiento de que para el ejercicio efectivo de la libertad es necesario garantizar niveles igualitarios en los aspectos sociales y económicos, lo que presenta base teórica más apropiada para definir conceptualmente equidad.

De este modo, garantizando la libertad de elección y compensando las desigualdades entre las correspondientes capacidades básicas determinadas por factores biológicos, socioeconómicos y por la disponibilidad de oferta existente, sería posible asegurar la efectiva igualdad de oportunidades en la consecución del bienestar.

\section{El concepto de equidad en salud}

La preocupación con la conceptualización del término equidad se ha mantenido en el centro de las discusiones de distintas áreas del conocimiento, dando lugar a numerosos trabajos. No obstante, en razón del interés especial en la problemática de la política de salud, serán sintetizadas a la continuación fundamentalmente contribuciones contemporáneas efectuadas por autores que dan prioridad al estudio de la temática de la equidad relativa a esa política social.

Un primer tratamiento de este tema es la diferenciación establecida por distintos autores (Carr-Hill, 1994; Wagftaff \& Van Doorslaer, 1993; West \& Cullis, 1979), entre equidad horizontal - tratamiento igual para iguales - y equidad vertical - tratamiento desigual para desiguales.

La distinción entre equidad horizontal y vertical expone dos problemas cruciales: (a) cuáles son los aspectos relevantes para dimen- 
sionar, en cada caso, la igualdad o la desigualdad; (b) que se entiende por tratamiento (Culyer,1993).

Por ejemplo, parece razonable que dos personas con el mismo problema de salud deban recibir igual tratamiento (Equidad horizontal). Una, en cambio, por disfrutar de condiciones físicas superiores, o mejor estado nutricional, reacciona más rápidamente a la terapéutica. En este caso, la igualdad de tratamiento resulta en desigualdad de resultado. En consecuencia, habría sido más equitativo proporcionar mejores cuidados al paciente cuya capacidad de respuesta fuese presumiblemente peor (Equidad vertical) (Le Grand, 1988).

De este modo, un mismo caso puede ser entendido de formas diferentes en función de las variables que sean utilizadas para dimensionar la igualdad. A su vez, la igualdad de tratamiento puede, por ejemplo, ser dimensionada como igualdad en el consumo de servicios de salud (a través de los inputs), o a partir de la igualdad de los resultados esperados (centrándose en los outcome) (Culyer, 1993). De este modo, conforme las definiciones previas efectuadas por el evaluador, un mismo caso puede ser abordado en términos de equidad horizontal o vertical.

Al mismo tiempo, la diferenciación efectuada entre equidad horizontal y vertical puede también ser cuestionada por todos aquellos que consideran que no hay dos personas iguales ni dos situaciones idénticas. En este caso, la equidad horizontal perdería aplicabilidad.

Otros autores, como Jardanovski \& Guimarães (1993), han hecho diferente lectura de los conceptos de equidad horizontal y vertical, asociando la primera al tratamiento intranecesidades y la segunda la relativa a las internecesidades. La equidad horizontal es entendida como tratamiento igual para necesidades de salud iguales, considerando la existencia de necesidades diferentes según el género, edad o condiciones sociales. La equidad vertical busca el tratamiento "apropiadamente desigual" de necesidades de salud distintas, incorporando la cuestión de las prioridades, por ejemplo, entre acciones preventivas y cirugías reparadoras o entre tratamiento de pacientes con deficiencias renales crónicas y cobertura integral en programas de asistencia al parto.

Sin duda, la equidad vertical, entendida como la priorización internecesidades, sirve de sustento a propuestas actuales - como las de cobertura de paquetes mínimos de necesidades de salud - y parte de presupuestos cuestionables - como, por ejemplo, que las necesidades, bien sean individuales bien regionales, siempre responden al mismo orden de prioridad - creyendo que es posible efectuar una jerarquización general de las necesidades. Aun reconociendo que en la práctica, la escasez de recursos puede imponer límites a la cobertura de las necesidades existentes, la jerarquización de las mismas es variable que no forma parte del concepto de equidad per se (Almeida et al., 1999).

Otra distinción importante entre tipos de equidad es la efectuada por Turner (1986), que relaciona:

- igualdad ontológica;

- igualdad de oportunidades;

- igualdad de condiciones;

- igualdad de resultados.

La idea de que existe igualdad ontológica entre los seres humanos es propia de ciertas concepciones religiosas y tradiciones morales, y se fundamenta en la noción de igualdad ante Dios.

En lo que atañe que a la igualdad de oportunidades, Turner identifica la existencia de un criterio meritocrático y la consi dera un evidente legado de las Revoluciones Francesa y Americana. El alcance de las realizaciones personales depende del mérito individual, dejando aparentemente de lado distinciones de edad, sexo o riqueza. Aparentemente, porque resulta fácil demostrar la existencia de diferencias de oportunidades, cuando están basadas exclusivamente en el mérito entre individuos de distintos grupos sociales: personas que, por herencia, parten de situaciones más favorables tanto con relación a su nivel económico como a su posición social, hay más oportunidades que otras que no cuentan con la misma herencia.

Considera, por tanto, que es necesario tomar en cuenta las desigualdades existentes en los puntos de partida (condiciones/ handicaps) para que la igualdad de oportunidad tenga algún significado real. Como ejemplo de realización de estetipo de objetivo, se citan las políticas Welfarianas en algunos países de Europa. Para finalizar, Turner asocia la igualdad de resultados a las políticas socialistas que pretenden compensar, además de las desi gualdades de condiciones, las diferencias de talento y habilidad.

En el ámbito de la salud, específicamente, Julian Le Grand es uno de los autores que más contribuciones ha traído con relación a la temática de la equidad. Él distingue cinco tipos de equidad concernientes a la distribución de los gastos públicos:

a) aquel que objetiva la igualdad del gasto público per capita;

b) el que persigue la igualdad derendimiento, buscando compensar los rendimientos de las 
poblaciones de más baja renta mediante el gasto público con políticas sociales, obteniéndose, de esta manera, una equiparación entre los rendimientos de los diferentes grupos sociales; c) el que busca la igualdad de utilización, en que el gasto público deberá favorecer la igualdad en el consumo de los diferentes servicios; d) en lo que se refiere a la igualdad del gasto, para el cual los recursos públicos deberán ser colocados de forma tal que todos los individuos tengan el mismo gasto personal por unidad de servicio utilizado;

e) lo que tiene por finalidad la consecución de la igualdad en los nivel es desalud por medio de distribución del gasto público orientado para la obtención de iguales resultados (Le Grand, 1982).

Se puede percibir la falta de una conceptualización única del término equidad y la existencia de diferentes dimensiones del concepto de equidad, en esta tipología presentada por Le Grand, debiendo ser, en verdad, entendida como diferentes formas de operacionalidad. Desde otro punto de vista, es posible afirmar que, exceptuando las distribuciones establecidas a partir de la igualdad de gasto, las restantes, para una efectiva operacionalidad, deberán tener en cuenta factores relativos a la determinación biológica y social de las necesidades de la población, tratando desigual mente los desiguales. Otro aspecto importante en la clasificación presentada está relacionado con el papel intervencionista del Estado en el alcance de mejores condiciones equitativas, ya que se trabaja a partir de la distribución del gasto público en todos los casos.

En trabajos más recientes, Le Grand (1988) afirma que la mayoría de los tipos de equidad mencionados, por representar variaciones de la misma idea o objetivo, puede ser sintetizada en tres partes:

a) tratamiento igual para necesidades iguales; b) igualdad de acceso;

c) igualdad de salud.

Cabe destacar que, en este caso, la igualdad de acceso es entendida como igualdad de costos personales para la obtención de tratamiento, incluyendo variables como distancia de los servicios y tiempo de espera para el servicio de atención. Sin embargo, lo que es denominado por el autor como simple síntesis, puede ser visto como cambio implícito en el concepto de equidad, ya que la nueva clasificación, en todos los casos y en alguna medida, está vinculada a factores determinantes de las desigualdades de necesidades existentes.

A su vez, Artells (1983) y Mooney (1983) consideran que es posible, a partir de la biblio- grafia existente, diferenciar si ete formas distintas de definir operacionalmente equidad:

a) igualdad degastos per capita

b) igualdad de recursos per capita: que incorpora a la distribución, realizada en base poblacional, correcciones en función de las diferencias de precio observadas en cada región.

c) igualdad de recursos para necesi dades iguales: que establece que, para obtener distribuciones equitativas, deben ser tomadas en cuenta las diferentes necesidades sanitarias existentes, efectuando correcciones con base tanto en el perfil demográfico como en el epidemiológico. d) igualdad de oportunidad de acceso para necesidades iguales: que reconoce, además de las diferentes necesidades determinadas por el perfil demográfico y epidemiológico, la existencia de desigualdades en el costo social del acceso (por ejemplo, la distancia a los servicios).

e) igualdad de utilización para iguales necesidades: que considera no sólo la distribución de la oferta y los costes sociales, como otros factores condicionantes de la demanda.

f) igualdad de satisfacción de necesidades marginales: que parte del presupuesto de que las necesidades mantienen el mismo orden de prioridades en las diferentes regiones. De esta manera, la equidad sería alcanzada cuando un incremento o corte en los recursos, el aumento o la disminución en la cobertura de las necesidades, fuesen los mismos en todas las regiones.

g) igualdad en las condiciones de salud: que tiene por objetivo la igualdad en los indicadores de salud.

Como se puede observar, la tipología presentada por Le Grand está contemplada en la revisión realizada por Artells (1983) y Mooney (1983) y, en términos generales, caben los comentarios ya efectuados. Al mismo tiempo, cabe destacar que la igualdad de satisfacción de necesi dades marginales es equivalente al concepto de equidad vertical asumido por Jardanovski \& Guimarães (1993).

I mporta señalar también que, para distribuciones regionales de recursos, son consideradas apropiadas exclusivamente las tres primeras definiciones operacionales mencionadas: igualdad de gasto per capita, igualdad de recursos per capita e igualdad de recursos para necesidades iguales (Mooney, 1983).

A su vez, Travassos (1992) distingue entre "equidad en salud" y "equidad en la utilización de los servicios de salud", observando que no todos los factores determinantes de las desigualdades en los niveles de salud intervienen en la determinación de las desigualdades en el uso de los servicios de salud y que la obtención de la “igualdad en el uso de los servicios 
de salud" no asegura la "igualdad en los resultados"

Dando prioridad a la discusión de la igualdad en la utilización de los servicios de salud, Travassos elabora un esquema para el análisis de sus determinaciones basándose en dos suposiciones centrales:

1) las necesidades en el ámbito de la salud están socialmente determinadas;

2) el uso de los servicios de salud está determinado tanto por las necesidades de salud de la población como por las características de la oferta del mercado de servicios de salud (política de salud).

En este caso, además de la determinación biológica y social de las necesidades, están considerados factores relacionados con la efectiva puesta a punto de la política de salud, sus características organizacionales y gerenciales por ejemplo, oferta de servicios, composición en términos del mix público/ privado, estructura de financiamiento, formas de pago etc. - y también el nivel de conocimiento y experiencia de los profesionales, su ideología y grado de autonomía (Travassos, 1992; Travassos et al., 2000). La autora se refiere aquí a la posibilidad de que un profesional elija tratamientos diferentes para necesidades iguales en función del grupo social a que pertenece cada paciente. En otras palabras, la ideología podría determinar el tratamiento mejor para el "ciudadano de primera" y el tratamiento de menor calidad para personas de baja renta. De este modo, esta interpretación de "igualdad en el uso de los servicios de salud" resulta más amplia que la presentada en la tipología elaborada por Artells (1983) y Mooney (1983), ya que incorpora factores relacionados con la calidad de las consultas y servicios.

Se destaca que los enfoques efectuados por los diferentes autores representan grados diferentes de aproximación, que van desde las características más generales hasta los aspectos específicos de la equidad en salud, entre los cuales además existen interrelaciones que están sintetizadas en la Tabla 1.

Para finalizar, es importante destacar el planteamiento efectuado por Whitehead (1991, 1992) con relación a las desigualdades que deben ser objeto de intervención para la obtención de estados sanitarios equitativos. La autora, partiendo de la dimensión ética del concepto de equidad, entiende que las desigualdades que deben ser tenidas en cuenta son aquellas consideradas al mismo tiempo evitables e injustas. En este sentido, a partir de la identificación de los factores que provocan el origen de las desigualdades en el campo de la salud se es- tablece una clasificación de los mismos en términos de injusticias e evitables.

Existe un cierto consenso sobre algunos factores que no pueden ser considerados injustos, tales como:

- Variaciones biológicas naturales

- Comportamientos peligrosos libremente escogidos, como por ejemplo determinados tipos de deportes.

- Ventajas temporales en materia de salud de un grupo en relación con otro grupo, por adoptar primero un comportamiento favorable a la salud. Siendo que los otros grupos podrán adoptar los mismos comportamiento en un corto espacio de tiempo.

Y otros factores son efectivamente considerados como injustos y evitables:

- Comportamientos peligrosos para la salud en situaciones donde los individuos tienen poca elección en términos de modo de vida;

- Exposición a condiciones de vida y de trabajo insalubres o que generen estrés;

- Acceso insuficiente a los servicios de salud y a otros servicios públicos fundamentales.

Sintetizando, se puede decir que:

1) Todos los autores trabajan sobre equidad en el sentido de alcance de mayores niveles de "igualdad", diferenciando alternativas que, en algunos casos, van desde la igualdad de gastos per capita hasta la igualdad de resultados.

2) Incluso considerando que en la mayor parte de los trabajos existen contribuciones importantes para la construcción de una definición conceptual de 'equidad', se observa una centralización en las definiciones operacionales y en la elección de los factores más adecuados para su realización.

3) Existe un cierto consenso, explícito o implícito, con relación al tratamiento de las desigualdades consideradas injustas y evitables y en cuanto a la necesidad de intervención del Estado en el proceso distributivo.

De esta manera, tomando como base lo expuesto, equidad debe ser entendida como el principio que rige funciones distributivas, las cuales tienen por objetivo compensar o superar las desigualdades existentes, consideradas socialmente injustas y evitables. Por tanto, desde un punto de vista teleológico, equidad e igualdad son sinónimos. No existen propuestas equitativas que tengan por objetivo final el alcance de mayores desi gualdades. Puede ser este uno de los factores que motivan el uso indistinto de los términos equidad e igualdad.

Resultados equitativos, sin embargo, presuponen redistribuciones desigualitarias de recursos, producto de ajustes efectuados en función de los factores biológicos, sociales y polí- 
Tabla 1

Interrelaciones entre los conceptos de igualdad presentados por diferentes autores.

\begin{tabular}{lll}
\hline Tuner & Le Grand & Artells/Mooney \\
\hline Igualdad de oportunidad & $\begin{array}{l}\text { Igualdad de gasto per capita } \\
\text { Igualdad de gasto per capita }\end{array}$ & $\begin{array}{l}\text { Igualdad de gasto per capita } \\
\text { Igualdad de recursos per capita } \\
\text { Igualdad de satisfacción de } \\
\text { necesidades marginales }\end{array}$ \\
$\begin{array}{l}\text { Igualdad de oportunidades/ } \\
\text { condiciones }\end{array}$ & Igualdad de rendimentos & $\begin{array}{l}\text { Igualdad de recursos para } \\
\text { necesidades iguales } \\
\text { Igualdad de acceso }\end{array}$ \\
Igualdad de utilización \\
\hline
\end{tabular}

tico-organizacionales determinantes de las desigualdades existentes.

En el ámbito de la salud, se destaca como deseable la igualdad de oportunidades en el uso de servicios de salud, en el sentido de oportunidades y condiciones de Turner, e incorporando además de la igualdad en el acceso la igualdad en el tratamiento necesario y en la calidad del mismo.

En cuanto a lo que se dice, en relación más específicamente con la distribución geográfica equitativa de recursos financieros en el sector de salud, se debe recordar que es condición necesaria - pero no suficiente - para el alcance del tratamiento equitativo entre grupos sociales y entre individuos, y que, en función de lo expuesto, el criterio más apropiado es la "definición de recursos según necesidades", entendiéndose que las necesidades están determinadas por un conjunto de factores, entre los cuales sobresalen los biológicos y socio-económicos.

En ese sentido, distribuciones geográficas más equitativas de recursos financieros deberían efectuar redistribuciones en favor de las regiones con condiciones sanitarias y socioeconómicas más precarias, para las cuales corresponderían tantos por ciento superiores a los estimados en función del tamaño poblacional.

Experiencia internacional en la distribución de recursos financieros

Tomando como referencia el concepto de equidad en salud en general, y la distribución equitativa de recursos financieros en los sistemas de salud, en particular, serán analizadas experiencias realizadas en Inglaterra, España e Italia, países que han procurado efectuar la distribución interregional de los recursos financie- ros destinados al sistema de servicios de salud basándose en criterios técnicos, objetivando el alcance de un mayor padrón de equidad.

En este contexto, el caso de Inglaterra presenta particular relevancia no sólo por ser la experiencia más prolongada, sino que, esencialmente, por la discusión que ha suscitado tanto a partir de su propuesta inicial como de sus sucesivas reformulaciones. Cabe destacar que la forma distributiva, llevada a cabo a partir de 1976 en el Reino Unido y que continuó vigente hasta 1991 (Sheldon et al., 1993), fue adoptada por otros países y considerada base de discusión importante para la definición de formas distributivas más equitativas, inclusive España e Italia. I gualmente, la metodología aplicada en Inglaterra en ese período fue también utilizada para evaluar la equidad en la distribución geográfica de recursos en estudios referidos a otros países, tales como India, Gana, Francia, Holanda y Finlandia (Bevan, 1991).

\section{Inglaterra}

El sistema de salud inglés surgió en los moldes del seguro social, transformándose posteriormente en un sistema regido por principios vinculados a los modelos de seguridad social. Desde el inicio de siglo, Inglaterra ha avanzado, desdeel punto de vista legal, en el sentido de la universalización de la cobertura conquistada, por medio del decreto que, al mismo tiempo, nacionalizó los hospitales y estableció el financiamiento del sistema con recursos generales de Estado, autorizado en 1944, y en especial con la creación del Sistema Nacional de Salud (SNS), en 1948.

Los principios definidos como básicos para la construcción de un sistema más equitativo consistieron en una cobertura universal tanto 
para servicios asistenciales como preventivos; en proporción, en el financiamiento, en función de la capacidad de pago; en igualdad en la oportunidad de acceso a los servicios; y, por fin, en distribución geográfica equitativa de recursos físicos y financieros (Whitehead, 1994).

Sin embargo, a pesar de la adopción de un sistema de salud dirigido por los principios wel farianos, hasta la década de los 60, los recursos eran colocados por medio de una dinámica presupuesto incrementalista, cuyo efecto era la manutención de las desigualdades regionales existentes, verificándose que las regiones más favorecidas en la distribución de los recursos financieros no eran aquellas las más necesitadas (Culyer et al., 1981).

Identificada la necesidad de promover un padrón interregional más equitativo en la distribución de los recursos, se procuró desde el principio equilibrar la oferta de servicios mediante programas de inversión que tenían por objetivo igualar la relación camas / población entre las regiones. Una propuesta ambiciosa, que fue desarrollada lentamente en razón a la falta de recursos financieros disponibles (Maynard \& Ludbrook, 1983, apud Giraldes, 1987).

En un segundo momento, al inicio de la década de los 70, se puso en práctica la primera metodología para el cálculo de los recursos correspondientes a las actividades hospitalarias de cada región. Esta se aplicaba únicamente a la distribución de recursos de costeo y consideraba tres tipos de indicadores regionales: (a) demográficos - composición de la población según sexo y edad; (b) disponibilidad de camas disponibles y, (c) flujo interregional de casos (Mays, 1995).

No obstante, las distribuciones efectuadas en los años posteriores poco se diferenciaban de la tendencia antes observada básicamente en función de los criterios escogidos que expresaban sólo de forma marginal los diferentes perfiles de las necesidades (Gran-Bretaña, 1976).

Así, con el objetivo de realizar una propuesta para la distribución de los recursos financieros que favoreciese la obtención de igualdad de oportunidad de acceso para necesidades iguales (Bloor \& Maynard, 1995), se designó una comisión que presentó sus recomendaciones en el RAWP, realizado en 1976. El método formulado en el RAWP partió de algunos principios básicos: debería ser aplicado con vistas a distribuir el total de los recursos, incluidos los relativos a los médicos generalistas (General Practiti oners - GPs); debería ser definido un tratamiento diferenciado para las transferencias de costos y de capital, y determinados criterios específicos para cada ítem de gastos - interna- mientos no psiquiátricos, servicios ambulatorios, salud colectiva, ambulancias, internamientos psiquiátricos, internamientos de incapacitados mentales, administración -; y, finalmente, debería ser aplicable tanto para las regiones (Regional Health Authorities - RHA) como para áreas (Area Health Authorities-AHA).

Cabe destacar que, a pesar de la recomendación efectuada en la propuesta original del RAWP, los gastos relativos a los GPs - parte esencial del gasto en atención primaria- no fueron incluidas en el total a ser distribuido (Bloor \& Maynard, 1995), el que perpetuó un sistema fraccionado e imposibilitó el efectivo redimensionamiento equitativo del total de recursos financieros (Majeed et al., 1994). Las diferencias regionales de número de GPs per capita $\mathrm{y}$, en consecuencia, de los gastos correspondientes se han mantenido prácticamente constantes (Le Grand, 1993).

Al tratar diferenciadamente los distintos tipos de servicios - internamientos no psiquiátricos, servicios ambulatorios, salud colectiva etc. - se hizo necesario definir el volumen de recursos destinados a cada uno, cálculo efectuado a partir de los gastos observados, lo que significó consolidar la estructura existente y aceptarla como la más adecuada. A su vez, como el método debería ser aplicable a la división de los recursos destinados tanto a las regiones como a las áreas y distritos, la selección de los indicadores se vio restringida por la posibilidad de obtener y procesar los datos correspondientes a estos tres diferentes niveles administrativos.

En 1994, basándose en un modelo de demanda de servicios de salud formulado por Carr-Hill et al. (1994), se elaboró una nueva propuesta para la distribución interregional de los recursos financieros. Simulaciones efectuadas a partir de la nueva metodología distributiva, con datos relativos a 1992, mostraron que su utilización en el caso de los recursos destinados para internamientos no psiquiátricos determinaría una división interregional no muy diferente de la obtenida por medio de la fórmula RAWP, mientras que en lo referente a la utilización psiquiátrica permitiría la redistribución expresiva en favor de las regiones menos privilegiadas (Peacoc \& Smith, 1995).

En este trabajo, en función de la experiencia acumulada y divulgada, el análisis del caso inglés se centra en la metodología propuesta en el RAWP para recursos de costos del sistema de salud. 
- Metodología distributiva propuesta por el Resource Allocation Working Party

Como ya se ha mencionado, el objetivo del RAWP fue elaborar una propuesta para la distribución interregional de los recursos financieros que favoreciese el alcance de una “igualdad de oportunidades de acceso para necesidades iguales" (Bloor \& Maynard, 1995:2). Para esto, se trató separadamente la distribución de los recursos destinados al costeo de los servicios hospitalarios de los correspondientes a los gastos de capital. Es importante resaltar que la propuesta relativa a los gastos de capital nunca fue realizada.

\section{a) Distribución de los recursos de costos}

En la distribución de los recursos de costos, fueron determinados criterios específicos para cada ítem de gasto: internamientos no psiquiátricos, servicios ambulatorios, salud colectiva, ambulancias, internamientos psiquiátricos, internamientos de incapacitados mentales y gastos administrativos.

\section{b) Internamientos no psiquiátricos}

Para la obtención de los porcentajes distributivos correspondientes a cada región, la población discriminada por sexo y franja de edad fue corregida a partir de indicadores relacionados con el perfil demográfico y la mortalidad.

El primer ajuste efectuado fue en función del perfil demográfico. Con este objetivo, se calculó la utilización esperada a partir de la base poblacional de cada subgrupo (sexo/ edad) en la región correspondiente y de las tasas de utilización observadas para el respectivo subgrupo poblacional a nivel nacional, según la causa básica - en función de los capítulos de la Clasificación Internacional de Enfermedades (CID) (OMS, 1995) - excluyéndose las relativas a las enfermedades psiquiátricas (Capítulo V), que son tratadas por separado.

Tal procedimiento equivale a la presuposición de que existe, para cada subgrupo poblacional en las diferentes regiones, la misma tasa de utilización observada a nivel nacional. De esta forma, el total de utilización esperado para cada región está determinado por las características regionales dimensionadas en función del tamaño de la población y de las correspondientes estructuras de sexo y edad. A continuación, la expectativa de utilización para cada grupo poblacional, según los capítulos de la CID, debe ser corregida en función de alguna medida de morbilidad, entendiéndose que la misma seria el mejor proxy de necesidades.

EI RAWP, ante la falta de información con relación a la morbilidad y a la poca confiabilidad de aquella existente, optó por trabajar con mortalidad como proxy de morbilidad y, por tanto, de necesidades. En este caso, se utiliza la Standardised Mortality Ratios - SMRs como factor de corrección, definiéndola como la relación entre la mortalidad observada por sexo, edad y causa básica en cada región y la esperada en función de la correspondiente tasa nacional (Carr-Hill et al., 1994; Gran Bretaña, 1976).

Se destaca que la SMR no se aplica a la ponderación de la utilización estimada en el caso de las causas básicas referentes a las enfermedades de piel (Capítulo XII), puesto que no existe el mismo grado de correlación entre morbilidad y mortalidad, ni incluso para las relativas el embarazo, parto y atención al recién-nacido (Capítulo XI), para las cuales se considera que el ajuste efectuado en función de las diversas estructuras demográficas es suficiente para tener una idea de las dimensiones de las necesidades regionales.

Se nota que las SMRs, cuando son calculadas para cada subgrupo poblacional, expresan desigualdades entre la mortalidad observada y la esperada que no se refieren a la estructura demográfica según sexo y edad, sino a otros factores determinantes de las necesidades regionales.

Sintetizando, para cada subgrupo poblacional son efectuados los siguientes cálculos:

$$
\begin{aligned}
& \text { Utilización Esperada }{ }_{i, j, k} \text { en } \mathrm{R}=\text { Población } \\
& \mathrm{R}_{\mathrm{i}, \mathrm{j}} \text { *Tasa de utilización nacional } \mathrm{i}_{\mathrm{i}, \mathrm{j}, \mathrm{k}} \\
& \text { Valor base } \mathrm{R}_{\mathrm{i}, \mathrm{j}, \mathrm{k}}=\text { Utilización esperada } \mathrm{R}_{\mathrm{i}, \mathrm{j}, \mathrm{k}} \\
& \text { *SMR R } \mathrm{S}_{\mathrm{i}, \mathrm{j}, \mathrm{k}} \\
& \mathrm{R}=\text { Región } \\
& \mathrm{I}=\text { sexo } \\
& \mathrm{J}=\text { franja de edad } \\
& \mathrm{K}=\text { causa básica según el CID }
\end{aligned}
$$

Finalmente, para determinar los valores base distributivos, es necesario obtener los subtotales regionales y un total nacional resultantes, en el primer caso, de la suma de los valores calculados por el subgrupo poblacional y conforme a los capítulos de la CID en cada región $y$, en el segundo, de la suma de los subtotales regionales. La comparación entre los subtotales regionales y el total nacional permite establecer la distribución porcentual de recursos de costos para internamientos no psiquiátricos.

En caso de que sea posible, se recomienda realizar una corrección adicional en función del flujo de pacientes de una región para otra. 
c) Internamientos psiquiátricos e Internamiento de incapacitados mentales

En caso de los internamientos psiquiátricos, la población fue discriminada por sexo, franja edad y estado civil. Esta última variable fue introducida por considerarse, a partir del perfil de utilización observado, que las personas casadas están menos sujetas a las enfermedades mentales. La mortalidad no fue considerada variable apropiada para determinar los niveles de necesidades de este tipo de servicio, en escasísimas situaciones las enfermedades mentales constituyeron causa directa de muerte. El estado civil no fue tenido en cuenta en el caso delos internamientos de incapacitados mentales.

De este modo los porcentajes distributivos fueron calculados exclusivamente a partir de Ios totales de utilización estimados para cada región. También en este caso se consideró conveniente efectuar correcciones adicionales en función del flujo interregional de pacientes. Al final, se recomendó que fuese efectuado un ajuste capaz de reflejar diferencias en cuanto a la proporción de internamientos permanentes o con varios años de duración.

\section{d) Servicios ambulatorios}

El procedimiento utilizado para el cálculo de los valores regionales para el costeo de las atenciones ambulatorias es similar al efectuado para internamientos no psiquiátricos. La utilización de ambulatorios esperada para cada subgrupo poblacional en función de las tasas nacionales es corregida a partir de la correspondiente SMR.

Cabe destacar que, en este caso, no fue efectuada la abertura por capítulo de la CID, dada la falta de información que se encontraba discriminada acerca de la utilización. En consecuencia, también las SM Rs fueron calculadas exclusivamente según sexo y edad.

Los val ores base distributivos y los correspondientes porcentajes son igualmente calculados a partir de la suma de subtotales regionales y de su comparación con el total nacional.

\section{e) Servicios de salud colectiva}

En esta situación, la población fue discriminada según franjas de edad sin existir distinción por sexo, y la utilización estimada para cada franja de edad fue corregida a partir de las respectivas SMRs. Los porcentajes distributivos son calculados a partir de los subtotales regionales, de la misma forma que en los casos anteriores. f) Servicios de ambulancia

Estudios demostraron que las variables edad y sexo no tenían poder explicativo en lo que concierne a la necesidad desplazamiento. Por tanto, los totales distributivos fueron obtenidos a partir de la población corregida en función de las SMRs globales.

\section{g) Costos administrativos}

Estos recursos están referidos exclusivamente a los costos administrativos generados por los GPs - los recursos para la remuneración de los GPs, como ya se mencionó, se quedaron excluidos de la fórmula distributiva. Los gastos administrativos restantes se incluyen en la distribución correspondiente a cada tipo de servicio. Para la distribución de este gasto, se tomó como base el número de habitantes.

h) Total regional para recursos de costeo

La síntesis distributiva para la financiación del costeo de los servicios de salud es calculada mediante la media ponderada de las distribuciones obtenidas para cada tipo de servicio internamientos no psiquiátricos, atención en ambulatorios etc. Los factores de ponderación están determinados en función de la participación relativa de cada tipo de servicio en el total del gasto efectuado el año anterior. Para concluir, son propuestos dos ajustes adicionales sobre la síntesis distributiva, uno en función de los costos especiales de los servicios con actividades de enseñanza e investigación y otro, para compensar, aunque parcialmente, las desigualdades existentes entre los costes de las diferencias regionales.

Según Carr-Hill (1994), la metodología RAWP presentó la ventaja de estar fundada en principios claros y de fácil entendimiento, permitiendo la comprensión y concienciación sobre las desigualdades regionales existentes e imposibilitando poderosos argumentos que pretendían la obtención de beneficios especiales para determinadas regiones. Cabe destacar que, la utilización de la formula RAWP permitió una ubicación regional más equitativa de los recursos financieros destinados al costeo de la asistencia hospitalaria (Le Grand, 1993).

\section{España}

La reforma sanitaria española - cuyo proceso de formulación se inició al final de la década de los 70 - fue consolidada, desde el punto de vista legal, en 1986, en la Ley General de la Sani- 
dad (LGS), la cual, en coherencia con lo establecido en la Constitución de 1978, o sea, el derecho de todo ciudadano a la protección a la salud, creó el Servicio Nacional de Salud (SNS). Se previó que el financiamiento de este servicio debería provenir de recursos provenientes de ingresos generales del Estado, la necesidad de integración de las diferentes redes asistenciales existentes y la transferencia gradual de su gestión a las Comunidades Autónomas (CCAA), teniendo, como principios fundamentales, la universalidad, la equidad, la solidariedad, la participación y el control comunitario.

Examinando específicamente el objetivo de la equidad, cabe destacar que el mismo no fue reconocido explícitamente en la legislación española hasta la promulgación de la LGS (Rodríguez et al., 1992), en la cual se establece, en diversos artículos, que el acceso a los servicios de salud se debe dar en condiciones de igualdad efectiva para todos los ciudadanos, que la política de salud deberá estar orientada a la superación de los desequilibrios territoriales y sociales, que las normas de utilización de los servicios sanitarios serán iguales para todos y que la política de gasto sanitario consistirá en instrumento fundamental para la corrección de las desigualdades existentes (Rodríguez et al., 1992; Ruiz-Alvárez et al., 1988).

En lo que se refiere a la descentralización en favor de las CCAA, el proceso iniciado anteriormente a la LGS, había sido realizado hasta 1991 sólo en seis de las diecisiete CCAA existentes, las cuales, en conjunto, daban cobertura asistencial para el 58\% de la población, continuando centralizada la responsabilidad de los servicios correspondientes al restante de la población (Almeida, 1995; Carmona et al., 1992; Costas-i-Terrones, 1988; Yrigoyen, 1992).

Para la superación de las desigualdades territoriales, es decir, entre las diferentes CCAA, se ha optado por criterios distributivos diversos entre instancias descentralizadas o no.

En el caso de las CCAA con gestiones descentralizadas los recursos de la seguridad social destinados a la cobertura asistencial fueron examinados según la población protegida población total, menos población protegida por sistemas especiales: Mutua General de Funcionarios Civiles del Estado (MUFACE); Instituto Social de las Fuerzas Armadas (ISFAS); Mutua General Judicial (MUGEJU); Mutua Nacional de Previsión de la Administración Local (MUNPAL). Ya los recursos correspondientes a las CCAA todavía no incorporadas al proceso de descentralización continuaron siendo colocados por el presupuesto global definido en función del gasto histórico observado. Cabe destacar que la LGS previó un período de diez años para ajustar la diferencia observada entre el gasto histórico de los servicios transferidos y el total de los recursos calculados a partir del nuevo criterio per capita mencionado. En resumen, serían efectuadas correcciones anuales del $10 \%$. Esta forma de distribución de los recursos financieros utilizada como instrumento para corregir las desigualdades existentes entre las diferentes regiones (CCAA) fue ampliamente criticada por distintos autores. La objeción principal está referida a la adopción exclusiva del criterio población protegida para la distribución de los recursos, una vez que no son contempladas las diferentes necesidades regionales (Carmona et al., 1992a, 1992b; Coll-Cuota, 1988; Costa-i-Terrones, 1988; Ortún, 1988).

Fundamentándose en las críticas efectuadas y con el objetivo de subsidiar la efectiva puesta a punto de la política redistributiva coherente con los principios del SNS, en especial en lo que se refiere a la equidad y solidaridad, fueron elaboradas propuestas alternativas que incorporaron, como proxy de necesidades, variables referentes al cuadro de mortalidad e indicadores relativos a la oferta de servicios (Carmona et al., 1992a, 1992b).

Cabe destacar que, a pesar de la existencia de propuestas alternativas más apropiadas para el alcance de los principios enunciados en la legislación, la entrega de los recursos destinados a las instancias descentralizadas continuó siendo efectuado según la estimación de la población protegida.

Así, España, a pesar de establecer en su legislación que el acceso a los servicios desalud se debe dar en condiciones de igualdad efectiva para todos los ciudadanos y que la política de salud debe estar orientada a la superación de las desigualdades existentes, al efectuar la división regional de los recursos a partir exclusivamente del concepto 'población protegida' ciertamente más equitativo que la simple distribución per capita - se limitó a dimensionar para cada región la población usuaria del Sistema Nacional de Salud, sin considerar diferencias en las necesidades relativas.

\section{$\underline{\text { Italia }}$}

Hasta la década de los 70, el modelo italiano de políticas sociales, en general, y de salud, en particular, se caracterizaba por la alta fragmentación y heterogeneidad institucional. En el campo específico de la salud, el sistema asistencial estaba configurado de modo predominante por las mutualidades, organizadas por categoría ocupacional, que ofrecían cobertura 
a cerca del 93\% de la población, y su principal fuente de financiamiento era la contribución obligatoria de trabajadores y empresas calculada sobre la hoja salarial. La transformación del modelo mutualista para otro, dirigido por principios de universalidad, equidad y solidariedad fue un proceso gradual, iniciado en los debates de la década de los 60, y que culminó con la creación del Servicio Sanitario Nacional (SSN), en 1978, por medio de la Ley $\mathrm{n}$ o 833. De esta forma, en el inicio de la década de los 80 , se pasó a un sistema público universal, unificado y descentralizado en favor de los "Enti locali" (municipios), reconociendo el derecho de ciudadanía y preconizando que se asegurase, en sentido amplio, "tratamiento igual para necesidades iguales" (Buratti, 1987a:53) y, de forma específica, "igual dad de acceso a los servicios de salud a toda la población” (Almeida,1995:273).

Desde el punto de vista del financiamiento, las contribuciones - transformadas en impuestos específicos para la salud - se centralizan en el Fondo Sanitario Nacional (FSN). Esta centralización, inspirada tanto en la experiencia nacional del Fondo Nacional para la Asistencia Hospitalaria (FNAH), creado en 1975, mientras que en el sistema vigente en Inglaterra (Arcangeli, 1991), fue considerada como exigencia derivada de los objetivos explicitados en la reforma de 1978, en especial en lo que se refiere a la disminución de las desigualdades existentes entre regiones y al alcance de la igualdad de acceso (Arcangeli, 1991; Hanau, 1987). La incorporación de criterios distributivos que objetivaban disminuir las desigual dades existentes fue explicitada por primera vez en el Plano Sanitario Nacional -1980/ 1982. En ese documento, se definieron dos tipos de techos financieros: uno, fundamentado en los gastos históricos de cada región y otro, teórico, calculado en función de la población subdividida en tres grupos de edad - 0-12 años; 13-60 años; más de 60 años - y corregi da según indicadores específicos: mortalidad infantil, enfermedades profesionales y accidentes de trabajo y mortalidad de enfermos. El volumen de recursos determinados en función del gasto histórico sería considerado como punto de partida, y las diferencias existentes a razón del cálculo del techo teórico según los criterios arriba mencionados deberían ser superadas en un periodo de seis años (Buratti, 1987a). A su vez, ante los desequilibrios existentes en la oferta de servicios, los recursos de capital serían destinados exclusivamente a las regiones más desfavoreci das.

Cabe destacar que parte importante de los recursos, considerados como destinados a gastos predeterminados, deberían permanecer excluidos de la base redistributiva de los nuevos criterios y transferida según la base poblacional.

Esta forma de división se convirtió en objeto de numerosas críticas centradas fundamentalmente en los indicadores adoptados para dimensionar las necesidades, su forma de utilización y en el criterio per capita utilizado para repartir los recursos excluidos, por ser considerados predeterminados. En 1981, en un seminario realizado en Milán, fue discutida la forma de distribución vigente, destacándose que el gasto total definido para cada región no se diferenciaba mucho del resultante cuando era utilizado el criterio per capita. Se concluyó, en ese seminario, que se debería subdividir el gasto por tipo de función y distribuirlo con indicadores específicos (Buratti, 1987b). Sin embargo, los valores destinados efectivamente realizados hasta 1981, inclusive, respondieron a una lógica totalmente diferente de la explicitada en los planes y proyectos gubernamentales. Se puede decir que el criterio práctico para la distribución de los recursos consistió en el denominado "pie de lista", que significa, en otras palabras, financiar los gastos efectuados (Hanau, 1987).

En el año 1982, se distribuyeron el 79\% de los recursos apoyán dose en el gasto histórico, $20 \%$ en función de la población residente y un $1 \%$ destinado a programas específicos, como el hanseniano y la cura termal, basándose en el peso relativo de la población objetivo respecto al total de habitantes. La misma forma distributiva fue aplicada en el período 1983-1984, sin embargo el gasto histórico utilizado fue un $69 \%$ de los recursos y la población residente recibió el restante 30\% (Buratti, 1987b). Por consiguiente, la sistemática distributiva implementada en el período 1982-1984, basada fundamentalmente en el gasto histórico observado en cada región, fue ampliamente criticada dada su incoherencia con el espíritu de la reforma sanitaria explicitado en la legislación de 1978.

En 1985 se adoptó la nueva forma distributiva para la determinación de los techos financieros regionales que, por un lado, tenían en cuenta las desigual dades existentes en las estructuras de edades regionales y, por otro, los costos relativos de los distintos servicios para las diferentes poblaciones.

En los últimos años de la década de los 80, en discrepancia con el espíritu de la ley de la reforma sanitaria, la repartición de los recursos fue efectuada fundamental mente en base poblacional, como recomendó o Rapporto Sanitario del documento Relazione Generalesulla 
Situazione Economica del Paese, de marzo de 1987, en el que se afirma que el indicador más apropiado para la repartición del FSN es la población residente (Quirino, 1991).

Por fin, Ia llamada “contrarreforma”, realizada en 1992, propuso una distribución en función de las contribuciones recaudadas en cada región, la compensación de los posibles déficit operacionales por parte de las instancias locales y la cobertura de "niveles uniformes de asistencia" o, en otras palabras, la definición de un "paquete básico" de servicios de salud (Almeida, 1995).

Así, se puede decir que, a pesar de las tentativas efectuadas para realizar distribuciones financieras tendientes a disminuir las desigualdades regionales, la contrarreforma propuso una repartición basada en la capacidad de recaudación de cada instancia local, forma de colocación que necesariamente tiende a consolidar las desigualdades existentes.

La experiencia internacional

y la distribución de recursos

financieros en Brasil

Es necesario destacar que, con distintos grados de explicitación, la equidad en el sistema de salud en los tres países estudiados está, desde el punto de vista legal, orientada por la igualdad de oportunidad de acceso para necesidades iguales, siendo operativa de distintas formas. No obstante, resulta obvio que la distribución interregional de recursos financieros, por más equitativa que sea, nunca podrá asegurar "igualdad de oportunidad de acceso para necesidades iguales", ya que su alcance estará también determinado por otros factores, como, por ejemplo, colocaciones intrarregionales.

En el caso específico de los países analizados, se puede decir que las formas distributivas fueron orientadas, de acuerdo con las clasificaciones realizadas por Artell (1983) y Mooney (1983), por la igualdad de recursos para necesidades iguales en Inglaterra y, en parte de los recursos financieros, en determinados períodos en Italia, o la si mple igualdad de gastos per capita, en España y, parcialmente en Italia. En algunos casos, en la práctica, las distribuciones estuvieron determinadas por los gastos históricamente observados o por los gastos efectivamente realizados (España e Italia).

Con relación a la aplicabilidad de cada experiencia en el caso brasileño, es importante señalar, por un lado, que la igualdad de gasto per capita - que representa la ventaja de la simplificación de su cálculo dado el nivel de desigualdades regionales existentes - no sería apropiada para la consecución de una colocación interregional más equitativa en Brasil.

No obstante, es necesario señalar que el criterio de población protegida llevado a cabo en España gana una especial relevancia particular en el caso brasileño, en que una importante parte de la población cuenta con seguros privados de salud. Ciertamente, distribuir recursos en función del criterio 'población protegida', en vista de la concentración de las poblaciones beneficiarias de los mencionados seguros en los estados de las regiones más favorecidas, permitiría una ubicación más equitativa, en especial delante de un cuadro de recursos escasos. Cabe destacar que la exclusión de la población protegida por seguros privados de salud estaría limitada al cálculo distributivo, garantizando la atención a través del Sistema Único de Salud (SUS) para toda la población, siendo que los gastos efectuados por el SUS con servicios prestados a beneficiarios de sistemas privados deberían ser resarcidos financieramente por la correspondiente empresa aseguradora.

A su vez, la metodología aplicada en Italia para la distribución de recursos en función de criterios de necesidades basados en indicadores específicos - como la mortalidad infantil, enfermedades profesionales y accidentes de trabajo, y la mortalidad en enfermos - representaría problemas relativos a la utilización de la tasa de enfermedades profesionales y accidentes de trabajo, ya que la misma podría no ser representativa en un país con aproximadamente $50 \%$ de su población económicamente activa insertada en el mercado informal de trabajo, especialmente considerando que ese mercado tiene importancia relativa diferente en las diversas categorías ocupacionales. Al mismo tempo, se destaca que, en la metodología inglesa, están contemplados los otros indicadores relacionados con el cuadro de mortalidad.

En lo que se refiere a la metodología aplicada en Inglaterra (RAWP) es importante resaltar que, si ella tuvo sensibilidad suficiente para dimensionar necesidades relativas en una sociedad con un esquema de desigualdades menos significativas que las observadas en el caso brasileño, ciertamente puede encargarse de la evaluación interregional o interestatal en Brasil.

En el caso brasileño, en la reforma sanitaria llevada a cabo a partir de la Constitución de 1988, el concepto de equidad está implícito en la universalidad de la cobertura y de la atención, así como en la uniformidad y equivalencia de los beneficios y servicios de salud para las poblaciones urbanas y rurales. De la nueva 
Carta Constitucional (1988) y de la legislación sectorial correspondiente, que creó el SUS, se infiere que el concepto de "igualdad deoportunidades de acceso a los servicios de salud se refiere a la igualdad de oportunidades para necesi dades iguales" (Almeida et al., 1999:5) e implica una discriminación positiva compensadora de las desigualdades existentes en los condicionantes del estado de salud de la población, considerados socialmente injustos. Mientras, el texto constitucional, en lo que se refiere a la Seguridad Social en general y a la Salud en particular, permite diferentes interpretaciones respecto a la igualdad de oportunidades.

El proceso de descentralización realizada desde los años 90, como eje central de la puesta a punto del SUS, ha redefinido la forma de ubicación de recursos financieros, pero se puede decir que poco se ha avanzado en el desarrollo de metodologías que apunten el alcance de mayores niveles de equidad en el sistema de salud (Almeida et al., 1999).

Simulaciones efectuadas para el caso brasileño, tomando como base informaciones relativas al año de 1994, mostraron que si los recursos financieros del MS destinados al costeo de Ios servicios de salud hubiesen sido distribuidos a partir de la fórmula RAWP, la ubicación se modificaría de forma substanciosa. Serían efectuadas transferencias de las Regiones Sur, Sudeste y Centro-Oeste en favor de las Regiones Norte y Nordeste, tanto comparándolas con la distribución realizada por el nivel federal en 1994, como con la observada a partir del criterio poblacional (Tabla 2). La Región Norte tendría un aumento de 57,51\%, cuando se la compara con los recursos destinados por el MS, y el aumento correspondiente a la Región Nordeste sería de $41,12 \%$. Los estados que recibirían proporcionalmente menos recursos serían: Rio Grande do Sul (30,30\%); Rio de Janeiro $(-26,69 \%)$; Distrito Federal $(26,33 \%)$; São Paulo (-21,78\%); y Paraná (21,68\%) (Almeida et al., 1999; Porto, 1997).

Cabe destacar que en función de la magnitud del sub-registro en la mortalidad notificada la simulación fue realizada tomando como base la mortalidad corregida. Las correcciones efectuadas se basaron en la estimación de cobertura de registro de óbitos según la Unidad Federativa, desarrollada por Szwarcwald et al . (1997).

Es importante también señalar que, como apunta la experiencia internacional, esa redistribución de los recursos interregionales (o inter-estados) debería ser gradual. Esta observación gana especial relevancia en el caso brasileño, dada la magnitud de la diferencia entre la ubicación observada y las reparticiones estimadas a través de la adaptación del RAWP. Redistribuciones radicales podrían comprometer el funcionamiento de los servicios de salud en los estados que pasarían a contar con un volumen inferior de recursos. Al mismo tiempo, para la obtención de mayor equidad interregional, la redistribución de recursos financieros destinados al costeo de los sistemas de salud debería estar acompañada de programas de inversión que tuviesen por objetivo equilibrar interregionalmente la oferta existente (Almeida et al., 1999).

Finalmente, a partir de 1998, fueron definidos los cambios en las formas distributivas realizadas por el MS, especialmente en lo que se refiere al financiamiento de la atención básica ambulatoria, con la creación del PAB, cuyos resultados desde el punto de vista de la equidad ubicativa todavía deben ser evaluados. Esta nueva sistemática distributiva a pesar de igualar valores per capita para el financiamiento de actividades ambulatorias consideradas básicas, no tiene en cuenta las desi gualdades interregionales, tanto en lo que se refiere a las necesidades existentes, como a los model os asistenciales de las diferentes regiones (Almeida et al., 1999).

\section{Consideraciones finales}

La necesidad de alcanzar mayor nivel de equidad es uno de los objetivos que ha obtenido mayor consenso tanto en lo que se refiere a las políticas sociales, en general, como a las políticas de salud, en particular. Sin embargo, en muchos casos, su validez termina restringida a la definición formal de un derecho, ya que las políticas llevadas a cabo no garantizan su efectivo ejercicio.

Específicamente en el caso brasileño, los principios orientadores explicitados en el texto constitucional, conjuntamente con las directrices del SUS, y de los determinantes y condicionantes de las condiciones de salud enunciadas en la Ley Organica de Salud - LOS - (alimentación, vivienda, saneamiento básico, medio ambiente, trabajo, renta, educación, transporte, ocio, y acceso a los bienes y servicios esenciales) permite inferir que el concepto de igualdad de oportunidades de acceso a los servicios de salud se refiere a la igualdad de oportunidades en el sentido de la propuesta elaborada por Sen (1992) y a la igualdad de condiciones conforme se encuentra en la tipología de Tuner (1986), implicando una discriminación positiva compensatoria de las desigualdades 
Distribución porcentual de los recursos proporcionados por el Ministerio de Salud (MS), de la población y de los recursos financieros estimados según la metodología RAWP. Brasil, 1994.

\begin{tabular}{|c|c|c|c|c|c|}
\hline $\begin{array}{l}\text { Unidad } \\
\text { Federativa }\end{array}$ & $\begin{array}{c}\text { Recursos (1) } \\
\text { MS - } 1994\end{array}$ & $\begin{array}{c}\text { Población (2) } \\
1994\end{array}$ & $\begin{array}{c}\text { Distribución \% (3) } \\
\text { con Metodología } \\
\text { RAWP }\end{array}$ & $\begin{array}{l}\text { Diferencia } \\
\%(3) /(1)\end{array}$ & $\begin{array}{l}\text { Diferencia } \\
\%(3) /(2)\end{array}$ \\
\hline Rondônia & 0,79 & 0,84 & 1,11 & 41,03 & 31,94 \\
\hline Acre & 0,25 & 0,29 & 0,42 & 69,17 & 45,00 \\
\hline Amazonas & 1,12 & 1,48 & 1,72 & 53,18 & 16,29 \\
\hline Roraima & 0,21 & 0,16 & 0,22 & 6,78 & 38,18 \\
\hline Pará & 2,23 & 3,47 & 3,88 & 74,44 & 11,93 \\
\hline Amapá & 0,14 & 0,21 & 0,27 & 92,79 & 29,24 \\
\hline Tocantins & 0,54 & 0,64 & 0,67 & 25,34 & 4,96 \\
\hline Norte & 5,27 & 7,09 & 8,30 & 57,51 & 17,04 \\
\hline Maranhão & 2,97 & 3,36 & 4,79 & 61,48 & 42,64 \\
\hline Piauí & 1,68 & 1,75 & 2,01 & 20,05 & 15,04 \\
\hline Ceará & 4,08 & 4,31 & 4,74 & 16,06 & 9,93 \\
\hline Rio Grande do Norte & 1,43 & 1,65 & 2,06 & 44,02 & 25,02 \\
\hline Paraíba & 2,10 & 2,15 & 2,24 & 6,65 & 4,38 \\
\hline Pernambuco & 4,16 & 4,79 & 5,57 & 33,78 & 16,24 \\
\hline Alagoas & 1,52 & 1,72 & 2,28 & 49,65 & 32,66 \\
\hline Sergipe & 0,82 & 1,03 & 1,50 & 82,48 & 45,99 \\
\hline Bahia & 5,89 & 8,10 & 9,60 & 63,03 & 18,55 \\
\hline Nordeste & 24,66 & 28,86 & 34,81 & 41,12 & 20,61 \\
\hline Minas Gerais & 10,39 & 10,63 & 9,02 & $-13,15$ & $-15,15$ \\
\hline Espírito Santo & 1,39 & 1,78 & 1,69 & 21,68 & $-5,23$ \\
\hline Rio de J aneiro & 11,84 & 8,58 & 8,68 & $-26,69$ & 1,19 \\
\hline São Paulo & 23,54 & 21,61 & 18,41 & $-21,78$ & $-14,81$ \\
\hline Sudeste & 47,15 & 42,60 & 37,80 & $-19,83$ & $-11,27$ \\
\hline Paraná & 6,35 & 5,63 & 4,97 & $-21,68$ & $-11,65$ \\
\hline Santa Catarina & 2,70 & 3,10 & 2,73 & 1,11 & $-11,95$ \\
\hline Rio Grande do Sul & 7,04 & 6,16 & 4,91 & $-30,30$ & $-20,29$ \\
\hline Sur & 16,10 & 14,89 & 12,61 & $-21,63$ & $-15,29$ \\
\hline Mato Grosso do Sul & 1,25 & 1,22 & 1,24 & $-0,72$ & 1,42 \\
\hline Mato Grosso & 1,40 & 1,46 & 1,58 & 13,16 & 8,19 \\
\hline Goiás & 2,82 & 2,76 & 2,66 & $-5,54$ & $-3,50$ \\
\hline Distrito Federal & 1,36 & 1,11 & 1,00 & $-26,33$ & $-9,79$ \\
\hline Centro-O este & 6,82 & 6,55 & 6,48 & $-4,98$ & $-1,04$ \\
\hline
\end{tabular}

Fuente: Porto (1997).

RAWP = Resource Allocation Working Party.

existentes, dimensionadas a partir de variables relacionadas con los mencionados condicionantes.

Así, en función del concepto de equidad implícito en la igualdad de oportunidades de acceso a los servicios de salud preconizado en la legislación brasileña, la distribución geográfica de recursos financieros necesariamente debería ser efectuada procurándose obtener igualdad de recursos para necesidades iguales.
En este sentido, basándose en la experiencia internacional analizada, la metodología para la distribución geográfica que fue considerada más apropiada para el alcance de una igualdad de recursos para necesidades iguales, es la puesta en práctica en Inglaterra por medio de la fórmula RAWP, ya que incorpora ajustes en función de dos determinantes fundamentales de las necesidades de salud: el perfil demográfico y el epidemiológico. 
Los resultados obtenidos en la simulación de la metodología RAWP para el caso brasileño, cuando se compararon con la ubicación efectuada en 1994 por el MS, muestran que los principios enunciados en la legislación no estuvieron presentes en la distribución geográfica de recursos financieros.

Finalmente se destaca que, como apuntan diferentes estudios (Navarro, 1999; van Doorslaer et al., 2000) una distribución geográfica de recursos financieros más equitativa no es suficiente para el alcance del tratamiento equitativo entre grupos sociales.

\section{Referencias}

ALMEIDA, C. M., 1995. As Reformas Sanitárias nos Anos 80: Crise ou Transição. Tese de Doutorado, Rio de Janeiro: Escola Nacional de Saúde Pública, Fundação Oswaldo Cruz.

ALMEIDA, C. M.; TRAVASSOS, C.; PORTO, S. \& BAPTISTA, T., 1999. A Reforma Sanitária Brasileira: Em Busca da Equidade. Documento Técnico 17. Brasília: Organização Pan-Americana da Saúde/ Organização Mundial da Saúde.

ARCANGELI, L., 1991. La spesa sanitaria programmata: Determinaziona e ruolo del Fondo Sanitario Nazionale. In: Welfare State \& Redistribuzione. Gli Effetti della Spesa Sanitaria e Pensionistica in Italia Negli Anni '80 (R. Brunetta \& L. Tronti, ed.), pp. 221-239, Milano: Fraco Angeli.

ARTELLS, J., 1983. Notas sobre la consideración econômica de la equidad: Utilización y acceso. In: Planificación y Economía de la Salud en las Autonomías (J. Artells, org.), pp. 23-30, Madrid: Masson.

BEVAN, G., 1991. L'Equite dans I'Utilisation des Moyens deSoins - Question d'Atualite. Cahier SHS 3. Geneva: World Health Organization.

BLOOR, K. \& MAYNARD, A., 1995. Equity in Primary Care. Discussion Paper 141. York: Center for Health Econimics, University of York.

BURATTI, C., 1987a. La ripartizione del fondo sanitario nazionale di parte corrente fra le regione: Un bilancio delle prime esperienze e qualche proposta per il futuro. In: Problemi Finanziari del Servizio Sanitario Nazionale (G. Muraro, ed.), pp. 53-95, Milano: Fondazione Smith Kline.
BURATTI, C., 1987b. II servizio sanitario nazionale fra decentramento istituzionale e controllo centrale della spesa. In: Problemi Finanziari del Servizio Sanitario Nazionale(G. Muraro, ed.), pp. 131-181, Milano: Fondazione Smith Kline.

CARMONA, G.; GOMEZ, J. S.; MARTIN, J. M.; MARTIN-MORENO, J.; MOLINA, A.; RUIZ, D.; SANCHEZ, J. M. \& SANFRUTOS, N., 1992a. Criterios de asignación territorial de recursos sanitarios. In: XI Jornada de Economía de la Salud, Anales, pp. 123-145, Alicante: Asociación Española de Economía de la Salud.

CARM ONA, G.; MARTIN, J.; SANFRUTOS, N.; MARTIN, J. M.; SALVADOR, J.; MOLINA, A.; RUIZ, D. \& SANCHEZ, J. M., 1992b. Metodologia para un nuevo modelo de financiación sanitario simulacion y resultados. In: XI Jornada de Economía de la Salud Alicante, Anales, pp. 146-167, Alicante: Asociación Española de Economía de la Salud.

CARR-HILL, R. A., 1994. Efficiency and equity implications of the health care reforms. Social Science and Medicine, 9:1189-1201.

CARR-HILL, R.; HARDMAN, G.; MARTIN, S.; PEACOCKS, S.; SHELDON, T. \& SMITH, P., 1994. A Formula for Distributing NHS Revenues based on Small Area Use of Hospital Beds. York: Centre for Health Economics, University of York.

COLL-CUOTA, P., 1988. Demografia y gasto sanitario: El criterio de financiación de la L.G.S. In: VIII Jornada de Economia de la Salud, Anales, pp. 199206, Canarias: Asociación Española de Economía de la Salud. 
COSTAS-I-TERRONES, J. C., 1988. Equidad territorial en España. Condicionantes económicos e institucionais. In: VIII Jornada de Economia de la salud, Anales, pp. 207-226, Canarias: Asociación Española de Economía de la Salud.

CULYER, A. J., 1988. Inequality of health services is, in general, desirable. In: Acceptable Inequalities (D. G. Green, ed.), pp. 31-45, Health Unit Paper 3, London: Institute of Economic Affairs.

CULYER, A. J., 1993. Health, health expenditures, and equity. In: Equity in theFinanceand Delivery Care: An International Perspective (E. van Doorslaer, A. Wagftaff \& F. Rutten, ed.), pp. 299-319, Commission of the European Communities Health Research, Series 8, Oxford: Oxford Medical Publications.

CULYER, A. J.; MAYNARD, A. \& WILLLIAMS, A., 1981. Alternative systems of health care provision: An essay on motes and beams. In: A New Approach to the Economics of Health Care (M. Olson, ed.), pp. 137-151. Washington, DC: American Enterprise Institute.

DHSS (Department of Health and Social Security), 1976. Sharing Resources for Health in England: Report of the Resource Allocation Working Party. London: DHSS.

FLEURBAEY, M., 1995a. Equal opportunity or equal social outcome? Economics and Philosophy, 11: 25-55.

FLEURBAEY, M., 1995b. Equality and responsibility. European Economic Review, 39:683-690.

GIRALDES, M. R., 1987. Distribuição Eqüitativa da Despesa em Cuidados de Saúde Primários. Tese para Concurso de Professor na Escola Nacional de Saúde Pública. Lisboa: Universidade Nova Lisboa. (mimeo.)

GOROVITZ, S., 1979. John Rawls: Uma teoria da justiça. In: Filosofia Política Contemporânea (S. Gorovitz, org.), pp. 77-120, Brasília: Universidade de Brasília.

HANAU, C., 1987. La ripartizione territoriale e la regolazione della spesa sanitaria in a Italia dopo la riforma. In: Problemi Finanziari del Servizio Sanitario Nazionale (G. Muraro, ed.), pp. 23-53, Milano: Fondazione Smith Kline.

JARDANOVSKI, E. \& GUIM ARÃES, P. C., 1993. O desafio da equidade no setor saúde. Revista de Administração deEmpresas, mai/jun:38-51.

KLEIN, R., 1988. Acceptable inequalities. In: AcceptableInequalities (D. G. Green, ed.), pp. 1-20, Unit Paper 3, London: Institute of Economic Affairs.

LACOSTE, J., 1992. A Filosofia no Século XX. Campinas: Papirus.

LE GRAND, J., 1982. The Estrategy of Equility, Redistribution and Social Services. London: George Allem \& Unwin.

LE GRAND, J., 1988. Equidad, Salud y Atención Sanitaria. In: VIII Jornada de Economia de la Salud, Anales, pp. 53-69, Canarias: Asociación Española de Economía de la Salud.

LE GRAND, J., 1993. Equity in the distribution of health care: The British debate. In: Equity in the Finance and Delivery Care: An Internacional Perspective (E. van Doorslaer, A. Wagftaff \& F. Rutten, ed.), pp. 349-355, Comission of the European Communities Health Research, Series 8. Oxford: Oxford Medical Publications.
MAJEED, F. A.; CHATURVEDI, N.; READING, R. \& BEN-SHLOMO, Y., 1994. Equity en the NHS: Monitoring and promoting in primary and secondary care. BMJ, 308:1425-1432.

MAYS, N., 1995. Geographical resource allocation in the English Nacional Health Service. International Journal of Epidemiology, 24:134-142.

MOONEY, G. H., 1983. Equity in health care: Confronting the confusion. Effective Health Care, 1:179-185.

NAVARRO, V., 1999. Health and equity in the world in the era of "globalization". International Journal of Health Services, 29:215-226.

OMS (Organização Mundial da Saúde), 1995. Classificação Estatística Internacional de Doenças e Problemas Relacionados à Saúde, 10ạ Revisão. v. 1. São Paulo: Centro Colaborador da OMS para a Classificação de Doenças em Português.

ORTUN, V., 1988. La equidad en la actual política sanitaria española: De la evidencia a la acción. In: VIII Jornada de Economia de la salud, Anales, pp. 2130, Canarias: Asociación Española de Economía de la Salud.

PEACOCK, S. \& SMITH, P., 1995. The ResourceAllocation Consequences of the New NHS Needs FormuIa. Discussion Paper 134. York: Center for Heath Economics, University of York.

PINKER, R., 1988. Towards a mixed economy of welfare in health care. In: Acceptable Inequalities (D. G. Green, ed.), pp. 49-64, Health Unit Paper 3, London: Institute of Economic Affairs.

PORTO, S., 1997. Equidade na Distribuição Geográfica de Recursos em Saúde: Uma Contribuição para o Caso Brasileiro. Tese de Doutorado, Rio de Janeiro: Escola Nacional de Saúde Pública, Fundação Oswaldo Cruz.

QUIRINO, P., 1991. I problemi connessi con una piú equilibrata ripartizione del Fondo Sanitario Nazionale. In: Welfare State \& Redistribuzione. Gli Effetti della Spesa Sanitaria e Pensionistica in Italia Negli Anni '80 (R. Brunetta, \& L. Tronti, ed.), pp. 313-333, Milano: Franco Angeli.

RAWLS, J., 1985. Teoria de la Justiça. Méjico: Fondo de Cultura Económica.

RODRIGUEZ, M.; CALONGE, S. \& REÑE, J., 1992. Analisis de la (in)equidad en la financiación y la provisión de los servicios en el sistema sanitario español. In: XI Jornada de Economía de la Salud Alicante, Anales, pp. 134-167, Alicante: Asociación Española de Economía de la Salud.

RUIZ-ALVAREZ, J. L.; GARCIA-DEL-VAL, M. \& NEGRO-LOPEZ, M., 1988. La equidad en el reparto de los recursos sanitarios: El caso español. In: VIII Jornada de Economia de la Salud, Anales, pp. 171180, Canarias: Asociación Española de Economía de la Salud.

SEN, A., 1992. Inequality Reexamined. Oxford: Clarendon Press.

SHETDON, T.; SMITH, G. D. \& BEVEN, G., 1993. Weighting in the dark: Resource allocation in the new NHS. BMJ, 306:835-839.

SZWARCWALD, C. L.; LEAL, M. C.; CASTILHO, E. A. \& ANDRADE, C. L. T., 1997. Mortalidade infantil no Brasil: Belíndia ou Bulgária. Cadernos de Saúde Pública, 13:503-516.

TRAVASSOS, C., 1992. Equity in the Use of Private 
Hospital Contracted by a Compulsory Insurance Scheme in the City of Rio de Janeiro, Brazil, in 1986. Ph.D. Thesis, London: School of Economics and Political Science.

TRAVASSOS, C.; VIACAVA, F.; FERNANDES, C. \& ALMEIDA, C., 2000. Desigualdades geográficas e sociais na utilização dos serviços de saúde no Brasil. Ciência \& Saúde Coletiva, 5:133-150.

TURNER, B., 1986. Equality. London: Tavistock Publications.

van DOORSLAER, E.; WAGFTAFF, A.; van der BURG, H.; CHRISTIANSEN, T.; DE GRAEVE, D.; DUVHESNE, I.; GERTHAM, U.; GERFIN, M .; GEURTS, J.; GROSS, L.; HAKKINEN, U.; JOHN, J.; KLAVUS, J.; LEU, R.; NOLAN, B.; O'DONNEL, O.; PROPPER, C.; PUFFER, F.; SCHELLHORN, M.; SUNDBERG, G. \& WINKELHAKE, O., 2000. Equity in the delivery of health care in Europe and the US. Journal of Health Economics, 19:553-583.

WAGFTAFF, A. \& van DOORSLAER, E., 1993 Equity in the finance and delivery of health care: Concepts and definitions In: Equity in theFinance and Delivery os Care: An Internacional Perspective (E. van Doorslaer, A. Wagftaff \& F. Rutten, ed.), pp. 7-19, Comission of the European Communities Health Research, Series 8. Oxford: Oxford Medical Publications.
WEST, P. \& CULLIS, J., 1979. Introducción a la Economía dela Salud. Oxford: Oxford University Press.

WHITEHEAD, M., 1991. Concepts et Principes de I'Égalite des Chances en Matière de Santé. Copenhague: Bureau Régional de l'Éurope, Organisation Mondiale de la Santé.

WHITEHEAD, M., 1992. The concepts and principles of equity in health. International Journal of Health Services, 22:429-445.

WHITEHEAD, M., 1994. Equity issues in the NHS: Who cares about equity in the NHS? BMJ, 308: 1282-1291.

YRIGOYEN, P., 1992. Regimen financiero de las transferencias del Instituto Nacional de la a la Comunidad Autonoma de Galicia y a la Comunidad Foral de Navarra. In: XI Jornada de Economía de la Salud, Anales, pp. 243-256, Alicante: Asociación Española de Economía de la Salud.

Recibido el 1 de junio de 2001

Versión final presentada el 18 de septiembre de 2001

Aprobado el 29 de octubre de 2001 